\title{
Orbital Alveolar Soft Part Sarcoma
}

National Cancer Institute

\section{Source}

National Cancer Institute. Orbital Alveolar Soft Part Sarcoma. NCI Thesaurus. Code C156276.

An alveolar soft part sarcoma involving the orbit. It usually occurs in children. 